\title{
A Parameterized Splitting System and Its Application to the Discrete Logarithm Problem with Low Hamming Weight Product Exponents
}

\author{
Sungwook Kim and Jung Hee Cheon \\ Department of Mathematical Sciences and ISaC-RIM, \\ Seoul National University, Seoul, 151-747, Korea \\ $\{$ avell7, jhcheon\}@snu.ac.kr
}

\begin{abstract}
A low Hamming weight product (LHWP) exponent is used to increase the efficiency of cryptosystems based on the discrete logarithm problem (DLP). In this paper, we introduce a new tool, called a Parameterized Splitting System, to analyze the security of the DLP with LHWP exponents.

We apply a parameterized splitting system to attack the GPS identification scheme modified by Coron, Lefranc and Poupard in CHES'05 and obtain an algorithm of $2^{61.6}$ time complexity which was expected to be $2^{78}$. Also a parameterized splitting system can be used to solve the DLP with a LHWP exponent proposed by Hoffstein and Silverman in $2^{54.51}$ time complexity, that is smaller than $2^{59}$ in the recent Cheon-Kim attack.
\end{abstract}

Keywords: Discrete Logarithm Problem with Low Hamming Weight Product (LHWP) Exponents, Parameterized Splitting Systems.

\section{Introduction}

It is important to compute exponentiations efficiently in cryptosystems based on the DLP. One approach to achieve this is to choose an exponent of low Hamming weight. For example, the GPS identification scheme proposed by Girault 457. uses as a secret key a product of two integers having low Hamming weight 45:7. Hoffstein and Silverman suggested a use of exponent $x=x_{1} x_{2} x_{3}$, where each integer $x_{i}$ has very low Hamming weight 9]. But a use of low Hamming weight exponents may weaken the security.

The Heiman-Odlyzko algorithm [8] and the Coppersmith's splitting system [3 10 16] have been used to analyze the DLP with low Hamming weight exponents. The complexity of solving the DLP with the Coppersmith's splitting system is about the square root of the size of the key space when the exponent is a single integer. It can be regarded to be almost optimal since the DLP has the square root complexity in the generic model [14].

In [9], Hoffstein and Silverman proposed an attack against low Hamming weight product (LHWP) exponents. In 4, Coron, Lefranc and Poupard combined the above attack with the Coppersmith's splitting system and described an algorithm that can be applied when the order of a group is unknown. But the complexity of the attack is far from the square root of the size of the key space. 
Our Results: In this paper, we generalize the Coppersmith's splitting system into a parameterized splitting system and propose its construction. It can be used to show that given a bit string of length $n$, weight $t$ and a positive integer $t_{1}<t$, there exists a part of the string of length $n_{1}$ and weight $t_{1}$ where $\frac{n_{1}}{t_{1}} \approx \frac{n}{t}$.

We apply a parameterized splitting system to the private key of the GPS identification scheme [47] and the Hoffstein and Silverman's exponent 9] (originally designated for $2^{80}$ bit security). In [4, Coron, Lefranc and Poupard proposed an attack with $2^{52}$ complexity to recover the private key of the GPS identification scheme from CHES'04 and suggested a new private key which is claimed to have the security level of $2^{78}$. But our parameterized splitting system reduces them to $2^{47.7}$ and $2^{65.5}$, respectively, and its randomized version reduces them to $2^{43.5}$ and $2^{61.6}$, respectively. In 11, Cheon and Kim introduced the notion of rotationfree elements and proposed an attack of $2^{55.9}$ complexity to the Hoffstein and Silverman's exponent. By combining the parameterized splitting system and the concept of rotation-freeness, we reduce it further to $2^{54.51}$

Organization of the Paper: In Section 2, we briefly introduce the HeimanOdlyzko algorithm, the Coppersmith's splitting system and the rotation-free elements. In Section 3, we propose a parameterized splitting system and its application to the DLP of LHWP exponents. In Section 4, we analyze the complexity of the GPS identification scheme and the DLP with the Hoffstein and Silverman's exponent. Finally, we conclude in Section 5.

\section{Preliminaries}

Let $g$ be a generator of a group $G$ and $x$ is an integer. From now on, ord $g$ and $w t(x)$ denote the order of $g$ and the Hamming weight of $x$, respectively.

Shanks' Baby-Step Giant-Step [13] and Pollard's Rho algorithm [1] are representative algorithms for the DLP. Algorithms for the DLP with low Hamming weight exponents are variants of Shanks' Baby-Step Giant-Step. In this section, we introduce the Heiman-Odlyzko algorithm, the Coppersmith's splitting system and the rotation-free elements. In this section, we assume ord $g$ is known.

\subsection{The Heiman-Odlyzko Algorithm}

The Heiman-Odlyzko algorithm [8] was introduced by Heiman and Odlyzko independently. (In 8], Heiman remarked this algorithm was independently noticed by Odlyzko.) In this section, we sketch the Heiman-Odlyzko algorithm.

We use the notations from [16]. We regard the binary representation of

$$
x=\sum_{i=0}^{n-1} x_{i} 2^{i}
$$

as the vector

$$
x=\left(x_{0}, \ldots, x_{n-1}\right) .
$$


Then this set of vectors corresponds to

$$
\left\{i: x_{i}=1\right\} \subset \mathbb{Z}_{n} \text {. }
$$

The following two mappings, which are inverse to each other, express the above correspondence.

$$
\begin{array}{ll}
\text { set }: & \left\{0,1, \ldots, 2^{n}-1\right\} \rightarrow 2^{\mathbb{Z}_{n}}, \operatorname{set}\left(x=\left(x_{0}, \ldots, x_{n-1}\right)\right)=\left\{i: x_{i}=1\right\} \\
\text { val }: & 2^{\mathbb{Z}_{n}} \rightarrow\left\{0,1, \ldots, 2^{n}-1\right\}, \operatorname{val}(Y)=\sum_{i \in Y} 2^{i}
\end{array}
$$

Consider the following equation

$$
y=g^{x}=g^{x_{1}+x_{2}},
$$

where $t=w t(x)=w t\left(x_{1}\right)+w t\left(x_{2}\right), w t\left(x_{1}\right)=t_{s}$ and $\operatorname{set}\left(x_{1}\right) \cap \operatorname{set}\left(x_{2}\right)=\emptyset$.

From the above equation, we get

$$
y g^{-x_{1}}=g^{x_{2}}
$$

Now we compute $y g^{-x_{1}}$ for all $x_{1} \in \mathbb{Z}_{n}$ such that $w t\left(x_{1}\right)=t_{s}$ and build a lookup table that contains all the pairs $\left(y g^{-x_{1}}, x_{1}\right)$ and support an efficient search on the first component. Then we compute $g^{x_{2}}$ for each $x_{2}$ such that $w t\left(x_{2}\right)=t-t_{s}$ and look up the table until a collision is found.

Neglecting logarithmic factors, the time complexity of the Heiman-Odlyzko Algorithm is $O\left(\left(\begin{array}{c}n \\ t_{s}\end{array}\right)+\left(\begin{array}{c}n \\ t-t_{s}\end{array}\right)\right)$. Since we need store only either the left or the right hand side, the space complexity of the Heiman-Odlyzko Algorithm is $O\left(\min \left\{\left(\begin{array}{c}n \\ t_{s}\end{array}\right),\left(\begin{array}{c}n \\ t-t_{s}\end{array}\right)\right\}\right)$.

\subsection{The Coppersmith's Splitting System}

The Coppersmith's splitting system was introduced in 10, based on the idea from [2]. Later, Stinson gave a good description of it in [16]. We follow this description.

Definition 1. (The Splitting System)

Suppose $n$ and $t$ are even integers, $0<t<n 1 A(n, t)$-splitting system is a pair $(X, \mathcal{B})$ that satisfies the following properties.

1. $|X|=n$ and $\mathcal{B}$ is a set of $\frac{n}{2}$-subsets of $X$ called blocks.

2. For every $Y \subseteq X$ such that $|Y|=t$, there exists a block $B \in \mathcal{B}$ such that $|Y \cap B|=\frac{t}{2}$.

Remark. An $(n, t)$-splitting system is denoted by an $(N ; n, t)$-splitting system if it has $N$ blocks.

The existence of a splitting system follows from this construction: Suppose $X=\mathbb{Z}_{n}=\{0,1, \ldots, n-1\}, B_{i}=\left\{i+j \bmod n: 0 \leq j \leq \frac{n}{2}-1\right\}, \mathcal{B}=\left\{B_{i}: 0 \leq\right.$ $\left.i \leq \frac{n}{2}-1\right\}$. Then, $(X, \mathcal{B})$ is an $\left(\frac{n}{2} ; n, t\right)$-splitting system.

The Coppersmith's splitting system enables us to restrict to $\mathcal{B}$ the search space of $x_{1}$ and $x_{2}$ in Equation (1). Hence This algorithm requires $N\left(\begin{array}{l}\frac{n}{2} \\ \frac{t}{2}\end{array}\right)$ time complexity and $\left(\begin{array}{c}\frac{n}{2} \\ \frac{t}{2}\end{array}\right)$ space complexity.

\footnotetext{
${ }^{1}$ Stinson constructed the splitting system even for odd $n$ and $t$ in [16].
} 
A Randomized Algorithm. The randomized version of the above algorithm is summarized in [16], which is also due to [3]. The time complexity of the randomized version is $O\left(\sqrt{t}\left(\begin{array}{c}\frac{n}{2} \\ \frac{t}{2}\end{array}\right)\right)$ and the space complexity of the randomized version is $O\left(\left(\begin{array}{c}\frac{n}{2} \\ \frac{t}{2}\end{array}\right)\right)$.

\subsection{Rotation-Free Elements}

In 1], Cheon and Kim defined an equivalent relation $\sim$ on $\mathbb{Z}_{2^{n}-1}$ as follows:

$a \sim b$ if and only if there exists a non-negative integer $i$ such that $a=2^{i} b$.

The idea of Cheon and Kim's attack on LHWPs is to reduce the key search space by considering only one element from each equivalent class.

Since there is no known algorithm to generate such representatives efficiently, they suggested a use of the set of rotation-free elements which contains at least one representative for each equivalent class. The set is only little bit larger than the number of equivalent classes and easily generated.

The definition of rotation-free elements is as follows:

Definition 2. (Rotation-Free Elements [1])

An element $z \in \mathbb{Z}_{2^{n}-1}$ is called a rotation-free element if there is a $k$-tuple $\left(a_{1}, a_{2}, \ldots, a_{k}\right)$ for a positive integer $k$ satisfying

1. $a_{i} \geq a_{1}$ for $1 \leq i \leq k$.

2. $\sum_{i=1}^{k} a_{i}=n$.

3. $z=2^{n-1}+2^{n-1-a_{1}}+\cdots+2^{n-1-\left(a_{1}+a_{2}+\cdots+a_{k-1}\right)}$.

Let $n, k$ be positive integers with $k<n$ and $R F(n, k)$ be the number of rotationfree elements of weight $k$ in $\mathbb{Z}_{2^{n}-1}$. Then $R F(n, k)$ is given in [1] by

$$
R F(n, k)=\sum_{i=0}^{\left\lfloor\frac{n}{k}\right\rfloor-1}\left(\begin{array}{c}
n-2-k i \\
k-2
\end{array}\right) .
$$

\section{Parameterized Splitting Systems}

In this section, we construct a Parameterized Splitting System, that is a generalization of the Coppersmith's splitting system. In the Coppersmith's splitting system, given $Y \subset \mathbb{Z}_{n}$, the size of a block $B$ such that $|Y \cap B|=\frac{t}{2}$ is fixed to $\frac{n}{2}$. We show that the size of a block B can be flexible so that $|Y \cap B|=t_{s}$ and $|B|=\left\lfloor\frac{t_{s} n}{t}\right\rfloor$ for any $0 \leq t_{s} \leq t$. This flexibility yields an efficient algorithm for the DLP with LHWP exponents. 


\subsection{Parameterized Splitting Systems}

We start with the definition of parameterized splitting systems.

Definition 3. (Parameterized Splitting Systems)

Suppose $n$ and $t$ are integers such that $0<t<n$. For any $t_{s}$ such that $0 \leq t_{s} \leq t$, a $\left(N ; n, t, t_{s}\right)$-parameterized splitting system is a pair $(X, \mathcal{B})$ that satisfies the following properties.

1. $|X|=n$ and $\mathcal{B}=\left\{B \subset X:|B|=\left\lfloor\frac{t_{s} n}{t}\right\rfloor\right\}$.

2. $|\mathcal{B}|=N$.

3. For every $Y \subseteq X$ such that $|Y|=t$, there exists a block $B \in \mathcal{B}$ such that $|Y \cap B|=t_{s}$.

Remark. We may assume $0<t<\frac{n}{2}, 1 \leq t_{s} \leq \frac{t}{2}$.

The following Lemma 1 constructs an efficient parameterized splitting system.

Lemma 1. $X=\{0,1, \ldots, n-1\}, Y=\left\{y_{1}, y_{2}, \ldots, y_{t}\right\} \subset X$ such that $|Y|=$ t. Suppose $t_{s}$ is an integer such that $0 \leq t_{s} \leq t$. Let $B_{i}=\{i \bmod n, i+$ $\left.1 \bmod n, \ldots, i+\left\lfloor\frac{t_{s} n}{t}\right\rfloor-1 \bmod n\right\}, i=0,1, \ldots, n-1$. Then, there exists $i$ such that $\left|Y \cap B_{i}\right|=t_{s}$.

Proof. For each $y \in Y$, let $\nu(y)=\left\{i: y \in B_{i}, i=0,1, \ldots, n-1\right\}$. Then, $|\nu(y)|=\left\lfloor\frac{t_{s} n}{t}\right\rfloor$.

Let $M$ be $\frac{1}{n} \sum_{i=0}^{n-1}\left|Y \cap B_{i}\right|$. Since $Y \cap B_{i}=\bigcup_{y \in Y}\left(\{y\} \cap B_{i}\right)$ and if $y_{i} \neq y_{j}$, then $\left(y_{i} \cap B_{i}\right) \cap\left(y_{j} \cap B_{i}\right)=\emptyset$,

$$
\begin{aligned}
M & =\frac{1}{n} \sum_{I=0}^{n-1}\left|Y \cap B_{i}\right|=\frac{1}{n} \sum_{i=0}^{n-1}\left|\bigcup_{y \in Y}\left(\{y\} \cap B_{i}\right)\right|=\frac{1}{n} \sum_{i=0}^{n-1} \sum_{y \in Y}\left|\{y\} \cap B_{i}\right| \\
& =\frac{1}{n} \sum_{y \in Y} \sum_{i=0}^{n-1}\left|\{y\} \cap B_{i}\right|=\frac{1}{n} \sum_{y \in Y}|\nu(y)|=\frac{t}{n}\left\lfloor\frac{t_{s} n}{t}\right\rfloor .
\end{aligned}
$$

From $\frac{t_{s} n}{t}-1<\left\lfloor\frac{t_{s} n}{t}\right\rfloor \leq \frac{t_{s} n}{t}$,

$$
t_{s}-1<t_{s}-\frac{t}{n}=\frac{t}{n} \cdot\left(\frac{t_{s} n}{t}-1\right)<\frac{t}{n}\left\lfloor\frac{t_{s} n}{t}\right\rfloor=M \leq \frac{t}{n} \cdot \frac{t_{s} n}{t}=t_{s} .
$$

Suppose there doesn't exist $B_{i}$ such that $\left|Y \cap B_{i}\right|=t_{s}$. If $\left|Y \cap B_{i}\right|<t_{s}$ for all $i$, then $M \leq t_{s}-1$, which contradicts with Equation (2). If $\left|Y \cap B_{i}\right|>t_{s}$ for all $i$, then $t_{s}+1 \leq M$, which contradicts with Equation (2).

By the above discussions, there exists $B_{i}$ and $B_{j}$ such that $\left|Y \cap B_{i}\right| \leq t_{s}$ and $\left|Y \cap B_{j}\right| \geq t_{s}$. However, from the fact $\left|Y \cap B_{i}\right|-\left|Y \cap B_{i+1}\right| \in\{-1,0,1\},\left|Y \cap B_{k}\right|$ should be $t_{s}$ for some $k \in\{i \bmod n, i+1 \bmod n, \ldots, j-1 \bmod n, j \bmod n\}$, which contradicts with the assumption.

Therefore, there exists $B_{i}$ such that $\left|Y \cap B_{i}\right|=t_{s}$.

Theorem 1. Let $X=\{0,1, \ldots, n-1\}, B_{i}=\{i \bmod n, i+1 \bmod n, \ldots, i+$ $\left.\left\lfloor\frac{t_{s} n}{t}\right\rfloor-1 \bmod n\right\}, \mathcal{B}=\left\{B_{i}: 0 \leq i \leq n-1\right\}$. Then, $(X, \mathcal{B})$ is a $\left(n ; n, t, t_{s}\right)$ parameterized splitting system. 
A Randomized Version. For given $Y$ and $t_{s}$, Theorem 1 implies that if we try at most $n$ blocks, we can find some block $B$ such that $|Y \cap B|=t_{s}$. In a randomized version, we randomly choose $B \subset \mathbb{Z}_{n}$ such that $|B|=\left\lfloor\frac{t_{s} n}{t}\right\rfloor$ and check whether $|Y \cap B|=t_{s}$. Then the probability of success is

$$
p=\frac{\left(\begin{array}{c}
t \\
t_{s}
\end{array}\right)\left(\begin{array}{c}
n-t \\
\left\lfloor\frac{t_{s} n}{t}\right\rfloor-t_{s}
\end{array}\right)}{\left(\begin{array}{c}
n \\
\left\lfloor\frac{t_{s}}{t}\right\rfloor
\end{array}\right)} .
$$

Lemma 3 shows that the expected number of trials to find a good block $B$ such that $|Y \cap B|=t_{s}$ is $O(\sqrt{t})$. We require Lemma 2 from [16] to get Lemma 3 .

Lemma 2. Suppose that $n$ and $\lambda n$ are positive integers, where $0<\lambda<1$. Define

$$
H(\lambda)=\lambda \log _{2} \lambda-(1-\lambda) \log _{2}(1-\lambda)
$$

Then

$$
\frac{2^{n H(\lambda)}}{\sqrt{8 n \lambda(1-\lambda)}} \leq\left(\begin{array}{c}
n \\
\lambda n
\end{array}\right) \leq \frac{2^{n H(\lambda)}}{\sqrt{2 \pi n \lambda(1-\lambda)}} .
$$

Lemma 3. $p>\sqrt{\frac{\pi}{2}} \cdot \sqrt{\left(\frac{t_{s}}{t}-\frac{1}{n}\right)\left(1-\frac{t_{s}}{t}\right)} \cdot t^{-1 / 2} \geq \frac{\sqrt{\pi}}{4} t^{-1 / 2}$.

Proof.

$$
p=\left(\begin{array}{c}
t \\
t_{s}
\end{array}\right) \frac{\left(\begin{array}{c}
n-t \\
\left\lfloor\frac{t_{s} n}{t}\right\rfloor-t_{s}
\end{array}\right)}{\left(\begin{array}{c}
n \\
\left\lfloor\frac{t_{s} n}{t}\right\rfloor
\end{array}\right)}=\left(\begin{array}{c}
t \\
\lambda_{1} t
\end{array}\right) \frac{\left(\begin{array}{c}
n-t \\
\lambda_{2}(n-t)
\end{array}\right)}{\left(\begin{array}{c}
n \\
\lambda n
\end{array}\right)}
$$

where $\lambda_{1}=\frac{t_{s}}{t}, \lambda_{2}=\frac{\left\lfloor\frac{t_{s} n}{t}\right\rfloor-t_{s}}{n-t}$ and $\lambda=\frac{\left\lfloor\frac{t_{s} n}{t}\right\rfloor}{n}$.

From Lemma 2,

$$
\begin{aligned}
p & \geq \frac{2^{t H\left(\lambda_{1}\right)}}{\sqrt{8 t \lambda_{1}\left(1-\lambda_{1}\right)}} \cdot \frac{2^{(n-t) H\left(\lambda_{2}\right)}}{\sqrt{8(n-t) \lambda_{2}\left(1-\lambda_{2}\right)}} \cdot \frac{\sqrt{2 \pi n \lambda(1-\lambda)}}{2^{n H(\lambda)}} \\
& =\frac{2^{t H\left(\lambda_{1}\right)+(n-t) H\left(\lambda_{2}\right)}}{2^{n H(\lambda)}} \cdot \frac{\sqrt{2 \pi n \lambda(1-\lambda)}}{8 \sqrt{t(n-t) \lambda_{1}\left(1-\lambda_{1}\right) \lambda_{2}\left(1-\lambda_{2}\right)}} .
\end{aligned}
$$

Since $H(\lambda)$ is convex,

$$
t H\left(\lambda_{1}\right)+(n-t) H\left(\lambda_{2}\right) \geq n H(\lambda),
$$

hence,

$$
p \geq \frac{\sqrt{2 \pi n \lambda(1-\lambda)}}{8 \sqrt{t(n-t) \lambda_{1}\left(1-\lambda_{1}\right) \lambda_{2}\left(1-\lambda_{2}\right)}} .
$$

Since $0<\lambda_{i}<1$,

$$
\frac{1}{\sqrt{\lambda_{i}\left(1-\lambda_{i}\right)}} \geq 2
$$

for $i=1,2$, hence,

$$
p \geq \sqrt{\frac{\pi}{2}} \cdot \sqrt{\lambda(1-\lambda)} \cdot t^{-1 / 2}
$$


We may assume $1 \leq t_{s} \leq \frac{t}{2}$ and $2 \leq t \leq \frac{n}{2}$. From $\lambda=\left\lfloor\frac{t_{s} n}{t}\right\rfloor / n$, we have

$$
\lambda(1-\lambda)>\left(\frac{t_{s}}{t}-\frac{1}{n}\right)\left(1-\frac{t_{s}}{t}\right) \geq \frac{1}{8} .
$$

\subsection{The DLP with LHWP Exponents When the Order of $g$ Is Known}

Before detailing how parameterized splitting systems can be used, we review some known methods.

For an integer $x$, we denote by $|x|$ the bit-length of $x$. Let $X_{1}=\left\{x_{1}:\left|x_{1}\right|=\right.$ $\left.n_{1}, w t\left(x_{1}\right)=t_{1}\right\}$ and $X_{2}=\left\{x_{2}:\left|x_{2}\right|=n_{2}, w t\left(x_{2}\right)=t_{2}\right\}$. Consider $x=x_{1} x_{2}$, where $x_{1} \in X_{1}$ and $x_{2} \in X_{2}$.

As in 49, from the following equation

$$
y=\left(g^{x_{1}}\right)^{x_{2}}=h^{x_{2}},
$$

$x$ can be computed by repeating an algorithm for the DLP by $\left|X_{1}\right|$. So, the time complexity and the space complexity of the Heiman-Odlyzko algorithm are

$$
O\left(\left|X_{1}\right|\left(\left(\begin{array}{c}
n_{2} \\
t_{s}
\end{array}\right)+\left(\begin{array}{c}
n_{2} \\
t-t_{s}
\end{array}\right)\right)\right) \text { and } O\left(\min \left\{\left(\begin{array}{c}
n_{2} \\
t_{s}
\end{array}\right),\left(\begin{array}{c}
n_{2} \\
t-t_{s}
\end{array}\right)\right\}\right)
$$

respectively. To minimize the time complexity, $t_{s}$ should be $\left\lceil\frac{t_{2}}{2}\right\rceil$ or $\left\lfloor\frac{t_{2}}{2}\right\rfloor$. The time complexity and the space complexity of the parameterized splitting system are

$$
O\left(\left|X_{1}\right| \cdot n_{2}\left(\begin{array}{c}
\frac{n_{2}}{2} \\
\frac{t_{2}}{2}
\end{array}\right)\right) \text { and } O\left(\left(\begin{array}{c}
\frac{n_{2}}{2} \\
\frac{t_{2}}{2}
\end{array}\right)\right)
$$

respectively.

Another attack, which is also followed from [4], takes the trade-off between time and space. $y=g^{x_{1} x_{2}}$ can be converted into

$$
y^{x_{1}^{-1}} g^{-x_{3}}=g^{x_{4}},
$$

where $x_{2}=x_{3}+x_{4}$ and $\operatorname{set}\left(x_{3}\right) \cap \operatorname{set}\left(x_{4}\right)=\emptyset$. Note that $x_{1}^{-1}$ denotes the multiplicative inverse of $x_{1}$ modulo the order of $g$.

Put $w t\left(x_{3}\right)=t_{s}$. From the above equation, we find $x_{1}$ and $x_{2}$ by computing both sides and comparing them.

Therefore the time complexity and the space complexity of the HeimanOdlyzko algorithm are

$$
O\left(\left|X_{1}\right|\left(\begin{array}{c}
n_{2} \\
t_{s}
\end{array}\right)+\left(\begin{array}{c}
n_{2} \\
t-t_{s}
\end{array}\right)\right) \text { and } O\left(\min \left\{\left|X_{1}\right|\left(\begin{array}{c}
n_{2} \\
t_{s}
\end{array}\right),\left(\begin{array}{c}
n_{2} \\
t-t_{s}
\end{array}\right)\right\}\right)
$$

respectively. $t_{s}$ is an integer such that $0 \leq t_{s} \leq\left\lceil\frac{t_{2}}{2}\right\rceil$. Comparing to the first application, the time complexity is lower. 
The time complexity and the space complexity of the splitting system are

$$
O\left(\left|X_{1}\right| \cdot \frac{n_{2}}{2}\left(\begin{array}{c}
\frac{n_{2}}{2} \\
\frac{t_{2}}{2}
\end{array}\right)+\frac{n_{2}}{2}\left(\begin{array}{c}
\frac{n_{2}}{2} \\
\frac{t_{2}}{2}
\end{array}\right)\right)=O\left(\left|X_{1}\right| \cdot \frac{n_{2}}{2}\left(\begin{array}{c}
\frac{n_{2}}{2} \\
\frac{t_{2}}{2}
\end{array}\right)\right) \text { and } O\left(\frac{n_{2}}{2}\left(\begin{array}{c}
\frac{n_{2}}{2} \\
\frac{t_{2}}{2}
\end{array}\right)\right)
$$

respectively. Comparing to the first application, the efficiency of the time complexity is hardly improved.

In the case of the DLP with a single integer exponent of low Hamming weight, the splitting system appears to be more efficient than the Heiman-Odlyzko algorithm since one of the factors of the time complexity, $n_{2}$, is reduced to $\frac{n_{2}}{2}$ in the splitting system. But the splitting system fixes $t_{s}=\frac{t_{2}}{2}$ while the Heiman-Odlyzko algorithm is able to choose $t_{s}$ arbitrary. This difference yields the Heiman-Odlyzko algorithm carries out trade-off efficiently while the splitting system does not.

Now we propose a new algorithm using parameterized splitting systems, which takes the advantages from both of previous algorithms. From Section 3.1, for $t_{s} \in$ $\left[0,\left\lceil\frac{t_{2}}{2}\right\rceil\right]$, there exists a $\left(n_{2} ; n_{2}, t_{2}, t_{s}\right)$-parameterized splitting system $\left(\mathbb{Z}_{n_{2}}, \mathcal{B}\right)$. So, there is a block $B_{i} \in \mathcal{B}$ such that $\left|\operatorname{set}\left(x_{2}\right) \cap B_{i}\right|=t_{s}$. Let

$$
\operatorname{set}\left(x_{3}\right)=\operatorname{set}\left(x_{2}\right) \cap B_{i} \text { and } \operatorname{set}\left(x_{4}\right)=\operatorname{set}\left(x_{2}\right) \cap\left(\mathbb{Z}_{n_{2}}-B_{i}\right) \text {. }
$$

Then, we get the following equation

$$
y^{x_{1}{ }^{-1}} g^{-\operatorname{val}\left(\operatorname{set}\left(x_{2}\right) \cap B_{I}\right)}=g^{\operatorname{val}\left(\operatorname{set}\left(x_{2}\right) \cap\left(\mathbb{Z}_{n_{2}}-B_{i}\right)\right)} .
$$

From the above equation, we get Algorithm 1. The first part of Algorithm 1 is to compute and store all the values of the left-hand side. The second part of Algorithm 1 is to compute each value of the right-hand side and check if it is in the list from the first part.

Now we present Algorithm 1 and its randomized version.

Analysis: Algorithm 1 needs $\left|X_{1}\right| \cdot n_{2}\left(\begin{array}{l}\left\lfloor\frac{t_{s} n_{2}}{t_{2}}\right\rfloor \\ t_{s}\end{array}\right)$ exponentiations in the first part and $n_{2}\left(\begin{array}{c}n_{2}-\left\lfloor\frac{t_{s} n_{2}}{t_{2}}\right\rfloor \\ t_{2}-t_{s}\end{array}\right)$ exponentiations in the second part. In Algorithm 1, we can store $\left(\operatorname{val}\left(Y_{2, i}\right), g^{\operatorname{val}\left(Y_{2, i}\right)}\right)$ 's instead of $\left(x_{1}, \operatorname{val}\left(Y_{1, i}\right), y^{x_{1}{ }^{-1}} g^{-\operatorname{val}\left(Y_{1, i}\right)}\right)$ 's. In this case, we compute $y^{x_{1}^{-1}} g^{-\operatorname{val}\left(Y_{1, i}\right)}$ and find a collision. So, we store one of two sets which has smaller cardinality. Thus, the time complexity and the space complexity (neglecting logarithmic factors) are

$$
\begin{gathered}
O\left(\left|X_{1}\right| \cdot n_{2}\left(\begin{array}{c}
\left\lfloor\frac{t_{s} n_{2}}{t_{2}}\right\rfloor \\
t_{s}
\end{array}\right)+n_{2}\left(\begin{array}{c}
n_{2}-\left\lfloor\frac{t_{s} n_{2}}{t_{2}}\right\rfloor \\
t_{2}-t_{s}
\end{array}\right)\right) \text { and } \\
O\left(\min \left\{\left|X_{1}\right| \cdot n_{2}\left(\begin{array}{c}
\left\lfloor\frac{t_{s} n_{2}}{t_{2}}\right\rfloor \\
t_{s}
\end{array}\right), n_{2}\left(\begin{array}{c}
n_{2}-\left\lfloor\frac{t_{s} n_{2}}{t_{2}}\right\rfloor \\
t_{2}-t_{s}
\end{array}\right)\right\}\right),
\end{gathered}
$$

respectively. 

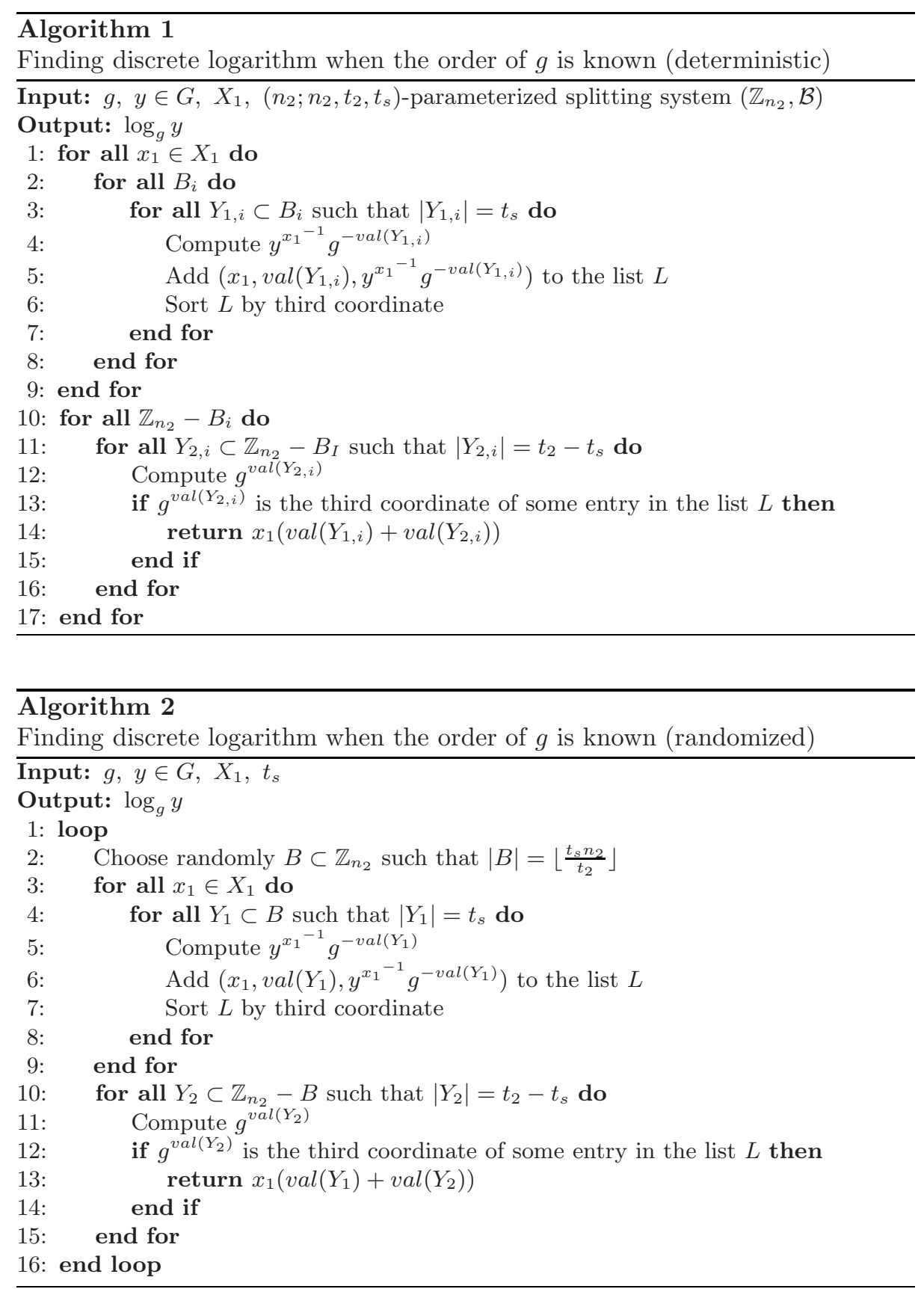
Lemma 3 implies that in about $\frac{4}{\sqrt{\pi}} t_{2}{ }^{1 / 2}$ iterations Algorithm 2 outputs $\log _{g} y$. And we only make $L$ for each $B$. Thus, if we count the number of group exponentiations, the time complexity and the space complexity are

$$
\begin{gathered}
O\left(\left|X_{1}\right| \cdot \sqrt{t_{2}}\left(\begin{array}{c}
\left\lfloor\frac{t_{s} n_{2}}{t_{2}}\right\rfloor \\
t_{s}
\end{array}\right)+\sqrt{t_{2}}\left(\begin{array}{c}
n_{2}-\left\lfloor\frac{t_{s} n_{2}}{t_{2}}\right\rfloor \\
t_{2}-t_{s}
\end{array}\right)\right) \text { and } \\
O\left(\min \left\{\left|X_{1}\right| \cdot\left(\begin{array}{c}
\left\lfloor\frac{t_{s} n_{2}}{t_{2}}\right\rfloor \\
t_{s}
\end{array}\right),\left(\begin{array}{c}
n_{2}-\left\lfloor\frac{t_{s} n_{2}}{t_{2}}\right\rfloor \\
t_{2}-t_{s}
\end{array}\right)\right\}\right),
\end{gathered}
$$

respectively.

\subsection{The DLP with LHWP Exponents When the Order of $g$ Is Unknown}

Recall the following equation in Section 3.2,

$$
y^{x_{1}^{-1}} g^{-x_{3}}=g^{x_{4}},
$$

If ord $g$ is unknown, $x_{1}^{-1}$ is not easy to compute from $x_{1}$ and so Equation (3) cannot be checked directly.

However, we can use Algorithm 1 or 2 from following trick from [4] and, earlier, proposed by Shoup [15]. Let

$$
\chi=\prod_{x \in X_{1}} x \text { and } \hat{g}=g^{\chi}
$$

From

$$
\left(y^{x_{1}{ }^{-1}} g^{-x_{3}}\right)^{\chi}=\left(g^{x_{4}}\right)^{\chi},
$$

we get

$$
y^{\prod_{x \in X_{1}-\left\{x_{1}\right\}} x} \cdot \hat{g}^{-x_{3}}=\hat{g}^{x_{4}},
$$

where $x_{2}=x_{3}+x_{4}$ and $\operatorname{set}\left(x_{3}\right) \cap \operatorname{set}\left(x_{4}\right)=\emptyset$.

To solving the DLP, we should perform the precomputation of $y^{\prod_{x \in X_{1}-\left\{x_{1}\right\}}}{ }^{x}$, $\hat{g}$ and $\hat{g}^{-1}$ and store them.

$\left\{y^{\prod_{x \in X_{1}-\left\{x_{1}\right\}^{x}}}: x_{i} \in X_{1}\right\}$ can be computed by the algorithm proposed by Coron, Lefranc and Poupard in [4]. According to the algorithm, $\left|X_{1}\right| \cdot \log _{2}\left|X_{1}\right|$ group exponentiations are necessary.

Therefore if we are able to learn $\hat{g}^{-1}$, we have Algorithm 3 and Algorithm 4.

Analysis: First, we analyze Algorithm 3. In Step 1, we perform $\left|X_{1}\right| \cdot \log _{2}\left|X_{1}\right|$ group exponentiations and store the results. There is no change of the time complexity and space complexity in Step 2. Therefore, the time complexity is

$$
O\left(\left|X_{1}\right| \cdot \log _{2}\left|X_{1}\right|+\left|X_{1}\right| \cdot n_{2}\left(\begin{array}{c}
\left\lfloor\frac{t_{s} n_{2}}{t_{2}}\right\rfloor \\
t_{s}
\end{array}\right)+n_{2}\left(\begin{array}{c}
n_{2}-\left\lfloor\frac{t_{s} n_{2}}{t_{2}}\right\rfloor \\
t_{2}-t_{s}
\end{array}\right)\right)
$$




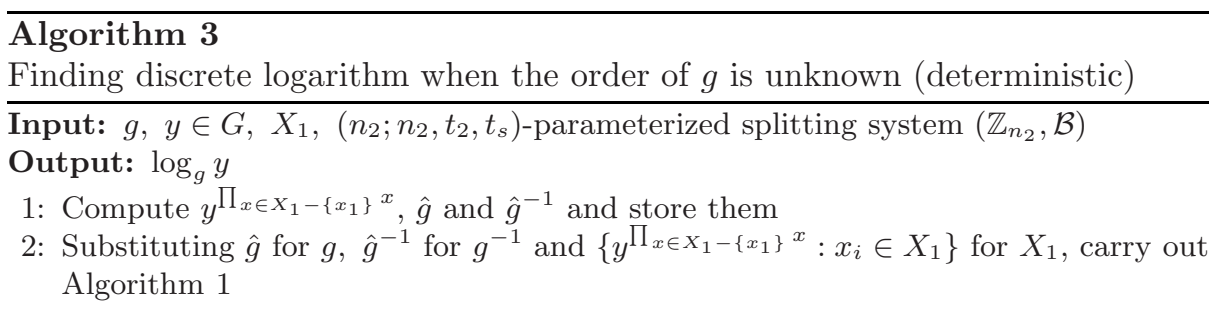

\section{Algorithm 4}

Finding discrete logarithm when the order of $g$ is unknown (randomized)

Input: $g, y \in G, X_{1}$

Output: $\log _{g} y$

1: Compute $y^{\prod_{x \in X_{1}-\left\{x_{1}\right\}^{x}}}, \hat{g}$ and $\hat{g}^{-1}$ and store them

2: Substituting $\hat{g}$ for $g, \hat{g}^{-1}$ for $g^{-1}$ and $\left\{y^{\Pi_{x \in X_{1}-\left\{x_{1}\right\}^{x}}}: x_{i} \in X_{1}\right\}$ for $X_{1}$, carry out Algorithm 2

and the space complexity is

$$
O\left(\left|X_{1}\right| \cdot \log _{2}\left|X_{1}\right|+\min \left\{\left|X_{1}\right| \cdot n_{2}\left(\begin{array}{c}
\left\lfloor\frac{t_{s} n_{2}}{t_{2}}\right\rfloor \\
t_{s}
\end{array}\right), n_{2}\left(\begin{array}{c}
n_{2}-\left\lfloor\frac{t_{s} n_{2}}{t_{2}}\right\rfloor \\
t_{2}-t_{s}
\end{array}\right)\right\}\right) .
$$

The best efficiency of the time complexity can be achieved when $\left|X_{1}\right|\left(\left\lfloor\frac{t_{s} n_{2}}{t_{s}}\right\rfloor\right) \approx$ $\left(\begin{array}{c}n_{2}-\left\lfloor\frac{t_{s} n_{2}}{t_{2}}\right\rfloor \\ t_{s}\end{array}\right)$. At this $t_{s},\left|X_{1}\right| \cdot \log _{2}\left|X_{1}\right|$ is negligible.

The only difference with Algorithm 3 is Step 2. Therefore, the time complexity is

$$
O\left(\left|X_{1}\right| \cdot \log _{2}\left|X_{1}\right|+\left|X_{1}\right| \cdot \sqrt{t_{2}}\left(\begin{array}{c}
\left\lfloor\frac{t_{s} n_{2}}{t_{2}}\right\rfloor \\
t_{s}
\end{array}\right)+\sqrt{t_{2}}\left(\begin{array}{c}
n_{2}-\left\lfloor\frac{t_{s} n_{2}}{t_{2}}\right\rfloor \\
t_{2}-t_{s}
\end{array}\right)\right)
$$

and the space complexity is

$$
O\left(\left|X_{1}\right| \cdot \log _{2}\left|X_{1}\right|+\min \left\{\left|X_{1}\right| \cdot\left(\begin{array}{c}
\left\lfloor\frac{t_{s} n_{2}}{t_{2}}\right\rfloor \\
t_{s}
\end{array}\right),\left(\begin{array}{c}
n_{2}-\left\lfloor\frac{t_{s} n_{2}}{t_{2}}\right\rfloor \\
t_{2}-t_{s}
\end{array}\right)\right\}\right) .
$$

Remark. We note that Algorithm 3 and 4 might output false answers. These errors come from the fact that the order of $\hat{g}$ of Equation (4) might be smaller than that of $g$. The worst case is that the order of $g$ is a divisor of that of $\hat{g}$. In this case, Equation (4) is an identical equation.

\section{Applications}

In this section, we attack the private keys of the GPS identification scheme [5]6]12 and the exponent proposed by Hoffstein and Silverman [9]. 


\begin{tabular}{|lll|}
\hline Prover & \multicolumn{1}{c|}{ Verifier } \\
choose $r \in\left[0,2^{R}[\right.$ & & \\
compute $W=g^{r} \bmod N$ & $\stackrel{W}{\longrightarrow}$ & \\
& $\stackrel{c}{ }$ & \\
choose $c \in\left[0,2^{k}[\right.$ & \\
check $c \in\left[0,2^{k}[\right.$ & $\stackrel{z}{\longrightarrow}$ & $\begin{array}{l}\text { check } z \in\left[0,2^{R}+2^{k+S}[\right. \\
\text { verify } g^{z} y^{c}=W\end{array}$ \\
\hline
\end{tabular}

Fig. 1. The GPS Identification Scheme

\subsection{Attacks on Private Keys of the GPS Identification Scheme}

We briefly introduce the GPS identification scheme.

GPS Identification Scheme. The GPS identification scheme, such as labelled by the NESSIE project, is an interactive protocol between a prover and a verifier which contains one or several rounds of three passes [7]. The GPS identification scheme is based on the DLP over $\mathbb{Z}_{N}{ }^{*}$. Precisely, when $g$ is an element of $\mathbb{Z}_{N}{ }^{*}$ of maximal order $m$, the GPS identification scheme is based on the DLP over $G=\langle g\rangle$, where ord $g$ is secret. When $y=g^{-x} \bmod N$, a private key of a prover is $x$ and public keys are $(N, g, y) . N$ is the product of two primes and the factorization of $N$ should be difficult.

There are four security parameters as follows:

I. $S$ is the binary size of $x$. Typically, $S=160$.

ii. $k$ is the binary size of the challenges sent to the prover and determines the level of security of the scheme.

iii. $R$ is the binary size of the exponents used in the commitment computation. It typically verifies $R=S+k+80$.

iv. $m$ is the number of rounds the scheme is iterated. Theoretically, $m$ is polynomial in the size of the security parameter. But, in practice, $m$ is often chosen equal to 1.

Private Keys of the GPS Identification Scheme. For the efficiency of the protocol, Girault and Lefranc proposed a private key $x$ as $x=x_{1} x_{2}$ in [7, where $x_{1}$ is a 19-bit number with 5 random bits equal to 1 chosen among the 16 least significant ones, $x_{2}$ is a 142-bit number with 16 random bits equal to 1 chosen among the 138 least significant ones in CHES'04.

Later in CHES'05, to strengthen the security, Coron, Lefranc and Poupard suggest the modified $x_{1}$ and $x_{2}$ in [4, where $x_{1}$ is a 30-bit number with 12 nonzero bits and $x_{2}$ is a 130 -bit number with 26 nonzero bits.

Attacks on Private Keys. We put $\left|X_{1}\right|=\left(\begin{array}{c}16 \\ 5\end{array}\right), n_{2}=138, t_{2}=16$ for private keys from [7] and $\left|X_{1}\right|=\left(\begin{array}{l}30 \\ 12\end{array}\right), n_{2}=130, t_{2}=26$ for private keys from [4]. Since $N$ is public we can easily compute $\hat{g}^{-1}$ of Algorithm 2, using the extended 
Euclidean algorithm. Before applying these private keys to Algorithm 3 and Algorithm 4, we note that when $t_{s}$ is chosen to guarantee the most efficient time complexity, the cost of precomputation is negligible.

Table 1 compares the complexities of recovering private keys from 7 and Table 2 for [4. The private key from [7] was broken in [4, which needs $2^{52}$ group exponentiations. But the parameterized splitting system and its randomized version reduce it further to $2^{47.7}$ and $2^{43.5}$, respectively.

Table 1. Private Keys from 7

\begin{tabular}{|c|c|c|}
\hline Method & Exponentiations & Storage \\
\hline \hline$[7]$ & $2^{52}$ & $2^{33}$ \\
\hline Ours (Algorithm 3), $t_{s}=7$ & $2^{47.7}$ & $2^{44.5}$ \\
\hline Ours (Algorithm 4), $t_{s}=7$ & $2^{43.5}$ & $2^{41}$ \\
\hline
\end{tabular}

Table 2 shows that the parameterized splitting system and its randomized version reduce the complexity of the DLP with the private key proposed in 4 from $2^{78}$ to $2^{65.5}$ and $2^{62.1}$, respectively.

Table 2. Private Keys from 4

\begin{tabular}{|c|c|c|}
\hline Method & Exponentiations & Storage \\
\hline \hline$[4$ & $2^{78}$ & $2^{43.9}$ \\
\hline Ours (Algorithm 3), $t_{s}=9$ & $2^{65.5}$ & $2^{63.1}$ \\
\hline Ours (Algorithm 4), $t_{s}=9$ & $2^{61.6}$ & $2^{59.2}$ \\
\hline
\end{tabular}

\subsection{Attacks on the Hoffstein and Silverman's Exponent}

The Hoffstein and Silverman's Exponent. Hoffstein and Silverman proposed a use of exponent $x=x_{1} x_{2} x_{3} \in \mathbb{Z}_{2^{1000}-1}$, where $x_{1}, x_{2}$ and $x_{3}$ are integers of $w t\left(x_{1}\right)=6, w t\left(x_{2}\right)=7$ and $w t\left(x_{3}\right)=7$ or $w t\left(x_{1}\right)=2, w t\left(x_{2}\right)=2$ and $w t\left(x_{3}\right)=11$ [9]. In the case of $w t\left(x_{1}\right)=6, w t\left(x_{2}\right)=7$ and $w t\left(x_{3}\right)=7$, all values of the Hamming weight are similar, hence, splitting of one's Hamming weight doesn't give advantages. So we focus on the case of $w t\left(x_{1}\right)=2, w t\left(x_{2}\right)=2$ and $w t\left(x_{3}\right)=11$.

Let $y=g^{x}$ for $x=x_{1} x_{2} x_{3}$ where $x_{i}$ 's are of weight $(2,2,11)$. Following the trick in [1], we rewrite $x$ as $x=2^{k} \bar{x}_{1} \bar{x}_{2} x_{3}$ where $0 \leq k<n$ and each of $\bar{x}_{i}$ are rotation-free elements in the same equivalent class with $x_{i}$ for each $i$. We further split $x_{3}$ by $x_{3}=x_{3}^{\prime}+x_{3}^{\prime \prime}$ where $x_{3}^{\prime}$ and $x_{3}^{\prime \prime}$ have weight 3 and 8 , respectively. Then we can find $x$ by checking the following equations:

$$
y^{2^{-k} \bar{x}_{1}^{-1} \bar{x}_{2}-1} g^{-x_{3}^{\prime}}=g^{x_{3}^{\prime \prime}} \text {. }
$$


In [1], Cheon and Kim modify $k$ so that $x_{3}^{\prime \prime}$ becomes rotation-free. Then the complexity for $n=1000$ is

$$
n \cdot R F(n, 2)^{2}\left(\begin{array}{c}
n-1 \\
3
\end{array}\right)+R F(n, 8) \approx 2^{55.2}+2^{54.5} \approx 2^{55.9} .
$$

On the other hand, if we combine the existence of a parameterized splitting system and the notion of the rotation-free, we get a little bit smaller complexity. When we split $x_{3}$, we apply the Theorem 1 to find a block $B$ such that $|B|=\left\lfloor\frac{3 n}{11}\right\rfloor$ and $\left|\operatorname{set}\left(x_{3}\right) \cap B\right|=3$. We write $\operatorname{set}\left(x_{3}\right) \cap\left(\mathbb{Z}_{n}-B\right)=\left\{s_{0}, s_{1}, \ldots, s_{7}\right\}$ and let $l_{i}$ be the number of elements of $\mathbb{Z}_{n}$ in $\left[s_{i}, s_{i+1}\right]$ for $i=0,1, \ldots, 7$, where we set $s_{8}=s_{1}$ and $\left[s_{7}, s_{1}\right]=\left\{s_{7}, \ldots, n-1,0, \ldots, s_{1}\right\}$. Suppose $l_{j}$ is the maximum of $l_{i}$ 's. Then, $l_{j}$ should be larger than $\left\lfloor\frac{3 n}{11}\right\rfloor$. We shift $x_{3}$ so that $s_{j}$ is placed at 0 .

From the above discussions, there exists an integer $k^{\prime}$ such that $2^{k^{\prime}} x_{3}=$ $x_{3}^{\prime}+x_{3}^{\prime \prime}$, where $x_{3}^{\prime}$ and $x_{3}^{\prime \prime}$ satisfy

1. $x_{3}^{\prime}$ is a string of length $n$ and weight 3 . If we write $\operatorname{set}\left(x_{3}^{\prime}\right)=\left\{a_{0}, a_{1}, a_{2}\right\}$ for $0<a_{0}<a_{1}<a_{2} \leq n-1$, then $a_{2}-a_{0}+1 \leq\left\lfloor\frac{3 n}{11}\right\rfloor$.

2. $x_{3}^{\prime \prime}$ is a string of length $n$ and weight 8 . If we write $\operatorname{set}\left(x_{3}^{\prime \prime}\right)=\left\{b_{0}, b_{1}, \ldots, b_{7}\right\}$ for $0=b_{0}<b_{1}<\cdots<b_{7} \leq n-1$, then $b_{i}-b_{i-1} \leq b_{1}$ and $\left\lfloor\frac{3 n}{11}\right\rfloor \leq b_{1}$.

To enumerate the number $N_{1}$ of $x_{3}^{\prime}$, we first fix $a_{0} \in[1, n-3]$ and then choose distinct $a_{1}, a_{2} \in\left[a_{0}+1, \min \left\{a_{0}-1+\left\lfloor\frac{3 n}{11}\right\rfloor, n-1\right\}\right]$. Hence

$$
N_{1}=\sum_{a_{0}=1}^{\left\lceil\frac{8 n}{11}\right\rceil}\left(\begin{array}{c}
\left\lfloor\frac{3 n}{11}\right\rfloor-1 \\
2
\end{array}\right)+\sum_{a_{0}=\left\lceil\frac{8 n}{11}\right\rceil+1}^{n-3}\left(\begin{array}{c}
n-1-a_{0} \\
2
\end{array}\right) .
$$

To enumerate the number $N_{2}$ of $x_{3}^{\prime \prime}$, we let $l_{0}=b_{1}, l_{i}=b_{i+1}-b_{i}$ for $i=1, \ldots, 6$ and $l_{7}=n-1-b_{7}$. Then, $N_{2}$ is the number of 8 -tuple $\left(l_{0}, \ldots, l_{7}\right)$ satisfying

1. $\sum_{i=0}^{7} l_{i}=n-1$.

2. $\left\lfloor\frac{3 n}{11}\right\rfloor \leq l_{0} \leq n-7$.

3. $1 \leq l_{i} \leq l_{0}$ for $i=1, \ldots, 6$ and $0 \leq l_{7} \leq l_{0}$.

First, we enumerate the number of solutions satisfying the above conditions when $l_{7} \neq 0$. Consider the following equation.

$$
\sum_{i=1}^{7} l_{i}=n-1-l_{0}
$$

This is the problem that how many solutions of positive integers the linear Diophantine equation (5) has when $1 \leq l_{i} \leq 0$ for $i=1, \cdots, 6$.

Given $l_{0}$, Let $A\left(l_{0}\right)$ be the set of solutions of Equation (5), $A_{i}\left(l_{0}\right)$ be the set of solutions when $l_{i}>l_{0}$ and $A_{i, j}\left(l_{0}\right)$ be the set of solutions when $l_{i}>l_{0}$ and $l_{j}>l_{0}$. Note that when $\left\lfloor\frac{3 n}{11}\right\rfloor \leq l_{0} \leq\left\lfloor\frac{n-2}{3}\right\rfloor$, only up to two values of $l_{i}, i=1, \cdots, 7$ can be larger than $l_{0}$, because otherwise, the sum of the others should be less than 0 . Similarly, when $\left\lfloor\frac{n-2}{3}\right\rfloor+1 \leq l_{0} \leq\left\lfloor\frac{n-2}{2}\right\rfloor$, only one value can be larger than $l_{0}$ 
and when $\left\lfloor\frac{n-2}{2}\right\rfloor+1 \leq l_{0} \leq n-7$, any value cannot be larger than $l_{0}$. Thus for given $l_{0}$, the number of solutions in the case of $l_{7} \neq 0$ is

$$
\begin{aligned}
N_{2,1}\left(l_{0}\right)^{\prime} & =\left|A\left(l_{0}\right)\right|-\left|\bigcup_{i=0}^{7} A_{i}\left(l_{0}\right)^{c}\right|=\left|A\left(l_{0}\right)\right|-\left\{\sum_{i=0}^{7}\left|A_{i}\left(l_{0}\right)\right|-\sum_{i \neq j}\left|A_{i, j}\left(l_{0}\right)\right|\right\} \\
& =\left(\begin{array}{c}
n-2-l_{0} \\
6
\end{array}\right)-\left\{7\left(\begin{array}{c}
n-2-2 l_{0} \\
6
\end{array}\right)-\left(\begin{array}{l}
7 \\
2
\end{array}\right)\left(\begin{array}{c}
n-2-3 l_{0} \\
6
\end{array}\right)\right\}
\end{aligned}
$$

when $\left\lfloor\frac{3 n}{11}\right\rfloor \leq l_{0} \leq\left\lfloor\frac{n-2}{3}\right\rfloor$. When $\left\lfloor\frac{n-2}{3}\right\rfloor+1 \leq l_{0} \leq\left\lfloor\frac{n-2}{2}\right\rfloor$,

$$
\begin{aligned}
N_{2,2}\left(l_{0}\right)^{\prime} & =\left|A\left(l_{0}\right)\right|-\left|\bigcup_{i=0}^{7} A_{i}\left(l_{0}\right)^{c}\right|=\left|A\left(l_{0}\right)\right|-\sum_{i=0}^{7}\left|A_{i}\left(l_{0}\right)\right| \\
& =\left(\begin{array}{c}
n-2-l_{0} \\
6
\end{array}\right)-7\left(\begin{array}{c}
n-2-2 l_{0} \\
6
\end{array}\right) .
\end{aligned}
$$

When $\left\lfloor\frac{n-2}{2}\right\rfloor+1 \leq l_{0} \leq n-7$,

$$
N_{2,3}\left(l_{0}\right)^{\prime}=\left|A\left(l_{0}\right)\right|=\left(\begin{array}{c}
n-2-l_{0} \\
6
\end{array}\right) .
$$

When $l_{7}=0$, the number of solutions $N_{2, i}\left(l_{0}\right)^{\prime \prime}, i=1,2,3$, can be computed in a similar way, i.e., 6 in each binomial is replaced to 5 .

Thus,

$$
\begin{aligned}
N_{2}= & \sum_{l_{0}=\left\lfloor\frac{3 n}{11}\right\rfloor}^{\left\lfloor\frac{n-2}{3}\right\rfloor}\left(N_{2,1}\left(l_{0}\right)^{\prime}+N_{2,1}\left(l_{0}\right)^{\prime \prime}\right)+\sum_{l_{0}=\left\lfloor\frac{n-2}{3}\right\rfloor+1}^{\left\lfloor\frac{n-2}{2}\right\rfloor}\left(N_{2,3}\left(l_{0}\right)^{\prime}+N_{2,3}\left(l_{0}\right)^{\prime \prime}\right) \\
& +\sum^{n-7}\left(N_{2_{3}}\left(l_{0}\right)^{\prime}+N_{2,3}\left(l_{0}\right)^{\prime \prime}\right) .
\end{aligned}
$$

Therefore, the total time complexity of the combined algorithm is

$$
n \cdot R F(n, 2)^{2} N_{1}+N_{2} \approx 2^{52.75}+2^{54.01} \approx 2^{54.51} .
$$

And the space complexity of the combined algorithm is about $2^{52.75}$.

\section{Conclusion}

In this paper, we have proposed a Parameterized Splitting System and its randomized version. Since a parameterized splitting system takes the advatages from both of the splitting system and the Heiman-Odlyzko algorithm, it gives an efficient algorithm for the DLP with LHWP exponents. 
Acknowledgements. The authors would like to thank Martijn Stam and the anonymous referees for valuable comments. The first author also would like to thank Namsu Jho for helpful discussions. This work was supported by the Korea Science and Engineering Foundation (KOSEF) grant funded by the Korea government (MOST) (No. R11-2007-035-01002-0).

\section{References}

1. Cheon, J., Kim, H.: Analysis of Low Hamming Weight Products; Discrete Applied Mathematics (to appear)

2. Coppersmith, D., Seroussi, G.: On the Minimum Distance of Some Quadratic Residue Codes. IEEE Trans. Inform. Theory 30, 407-411 (1984), MR 86c:94025

3. Coppersmith, D.: Private communication to Scott Vanstone (December 1997)

4. Coron, J., Lefranc, D., Poupard, G.: A New Baby-Step Giant-Step Algorithm and Some Application to Cryptanalysis. In: Rao, J.R., Sunar, B. (eds.) CHES 2005. LNCS, vol. 3659, pp. 47-60. Springer, Heidelberg (2005)

5. Girault, M.: Self-Certified Public Keys. In: Davies, D.W. (ed.) EUROCRYPT 1991. LNCS, vol. 547, pp. 490-497. Springer, Heidelberg (1991)

6. Girault, M., Poupard, G., Stern, J.: Some Modes of Use of the GPS Identification Scheme. In: 3rd Nessie Conference, Springer, Heidelberg (November 2002)

7. Girault, M., Lefranc, D.: Public Key Authentication with One Single (on-line) Addition. In: Joye, M., Quisquater, J.-J. (eds.) CHES 2004. LNCS, vol. 3156, pp. 413-427. Springer, Heidelberg (2004)

8. Heiman, R.: A Note on Discrete Logarithms with Special Structure. In: Rueppel, R.A. (ed.) EUROCRYPT 1992. LNCS, vol. 658, pp. 454-457. Springer, Heidelberg (1993)

9. Hoffstein, J., Silverman, J.: Random Small Hamming Weight Products with Application to Cryptography. Discrete Appl. Math. 130(1), 37-49 (2003)

10. Menezes, A., van Oorschot, P., Vanstone, S.: Handbook of Applied Cryptography, p. 128. CRC Press, Boca Raton (1997)

11. Pollard, J.: Monte Carlo Methods for Index Computation ( $\bmod \mathrm{p})$. Mathematics of Computation 32(143), 918-924 (1978)

12. Poupard, G., Stern, J.: Security Analysis of a Practical "On the Fly" Authentication and Signature Generation. In: Nyberg, K. (ed.) EUROCRYPT 1998. LNCS, vol. 1403, pp. 422-436. Springer, Heidelberg (1998)

13. Shanks, D.: Class Number, a Theory of Factorization and Genera. Proc. Symp. Pure Math. 20, 415-440 (1971)

14. Shoup, V.: Lower Bounds for discrete Logarithms and Related Problems. In: Fumy, W. (ed.) EUROCRYPT 1997. LNCS, vol. 1233, pp. 256-266. Springer, Heidelberg (1997)

15. Shoup, V.: Practical Threshold Signatures. In: Preneel, B. (ed.) EUROCRYPT 2000. LNCS, vol. 1807, pp. 207-220. Springer, Heidelberg (2000)

16. Stinson, D.: Some Baby-Step Giant-Step Algorithms for the Low Hamming Weight Discrete Logarithm Problem. Mathematics of Computation 71(237), 379-391 (2002) 